\section{First-in-class approval rate stays steady}

Year-to-year variation in drug approvals is driven by "addition-to-class" agents, reports the US Food and Drug Administration (FDA).

The lowdown: Annual approval numbers provide a quick and easy gauge of the health of the biotech and pharmaceutical industry, with year-to-year variations leading to hand-wringing or excitement. But like all top-line data, these numbers don't tell the full story. To better measure industry's ability to innovate, the FDA took a closer look at the new molecular entity (NME) approval rates and found we may be making much ado about stability. "The number of first-in-class drugs remained remarkably stable over 25 years, with an average of roughly eight new first-in-class drugs per year," wrote the FDA's Janet Woodcock and her colleagues (Health Affairs 32, 1433-1439; 2013).

To get to these numbers, Woodcock and her colleagues classified each of 645 NME approvals made between 1987 and 2011 as "first-in-class", "advance-in-class" (defined as a non-first-in-class product that receives a priority review designation) or "addition-to-class". When they plotted the results by year, they found that much of the variation in approval rateincluding a surge in the mid-1990s — can be traced back to the fortunes of addition-to-class products.

The authors also found some cause for optimism, noting that first-in-class drugs made up $39 \%$ of the total approvals during 2002 to 2011 , up from $27 \%$ of the total in the preceding 15 years.

Large pharmaceutical companies (defined as the top 25 companies based on US sales revenues in the respective year) generated $55 \%$ of the NMEs, and small companies generated the rest. Yet, despite producing fewer NMEs, small companies accounted for $53 \%$ of the first-in-class agents and only $40 \%$ of the addition-to-class agents.

Although the findings of stability are encouraging, the authors note that maintenance of the status quo may not be enough to sustain the industry. Indeed, research and development (RED) efficiency, as measured by how many new drugs are brought to market per billion US dollars in RED spend, has been falling steadily (Nature Rev. Drug Discov. 11, 191-200; 2012). As various initiatives are being implemented to slow the efficiency decline, better measures of industry's track record — including sophisticated analysis of NME approvals — should help us track whether progress is being made, the authors conclude.

\section{A comeback for anti-CD3s in type 1 diabetes?}

Teplizumab met its primary end point in an academic-led Phase II trial, a few years on from a programme-killing pivotal-trial failure. The lowdown: Type 1 diabetes is characterized by the progressive loss of self-tolerance to insulin-producing pancreatic islet $\beta$-cells, prompting drug developers to seek out immunomodulatory agents that can dampen the autoimmune response. Hopes for one such class of agent - monoclonal antibodies specific for CD3 - seemed to be dashed in 2010 when a Phase III trial of Lilly and Macrogenics' CD3-targeting teplizumab failed to meet its primary end point in the pivotal Protégé trial (Lancet 378, 487-497; 2011). Lilly subsequently discontinued its development of the drug, returning rights to Macrogenics. GlaxoSmithKline pulled the plug a year later on a competing agent, otelixizumab, licensed from Tolerx, when it too failed in a Phase III trial in the same indication. But results from a US National
Institute of Allergy and Infectious Diseases (NIAID) trial now suggest the tide could be turning.

Protégé investigator Kevan Herold, of Yale University, and his colleagues carried out a follow-up trial of teplizumab after noting a larger treatment effect associated with younger age, recruitment from the United States and early treatment in the Protégé data. Their NIAID-sponsored Phase II study — carried out in younger patients in North America - has now met its primary end point, giving a new boost to the anti-CD3 hypothesis (Diabetes http://dx.doi.org/10.2337/db13-0345; 2013).

The authors point out that type 1 diabetes is relatively easy to diagnose in North Americans and individuals there tend to have lower insulin requirements, lower glycated haemoglobin (HbA1c) levels and lower incidence of diabetic ketoacidosis, potentially explaining the geographical success.

The team also found that metabolic and immunological features - including low $\mathrm{HbA} 1 \mathrm{c}$ and insulin use at the time of enrolment - were significantly lower for responders than for non-responders.

"We are trying to launch a Phase III trial," Herold told Nature Reviews Drug Discovery. $\mathrm{He}$ adds that the drug has shown efficacy in five separate controlled trials, and he remains optimistic it can be approved for treatment of new-onset disease.

GlaxoSmithKline still lists otelixizumab as in development for rheumatoid arthritis.

\section{Improving animal research}

One study found evidence of bias in the literature on animal studies of neurological disease, while another investigated how the validity of animal research can be improved. The lowdown: The limitations and challenges of animal studies are well established.

Not only do biological differences between humans and animals muddy the interpretation of results, but reporting bias and shortcomings in experimental design can undermine the validity of new findings.

In an attempt to gauge the magnitude of the reporting bias problem, John loannidis, of Stanford University, and his colleagues analysed 4,445 animal studies of neurological diseases that had been synthesized into meta-analyses and looked for evidence that too many of the studies reported statistically significant results. Whereas with "reasonable assumptions about the plausible effect size" the authors expected that 919 of these 4,445 studies should have reported statistically significant results, they observed 1,719 such studies (PLoS Biol. 11, e1001609; 2013). "There are too many animal studies with statistically significant results in the literature of neurological disease," the authors conclude.

A separate study looked at what could be done to improve the validity of animal research. Dan Hackam, of the University of Western Ontario, and his colleagues reviewed the literature and found 26 guidelines offering 55 recommendations for how to minimize threats to animal data validity (PLoS Med. 10, e1001489; 2013). The most common recommendations included the use of power calculations to determine sample size, randomization and disease phenotype characterization. "By systematizing widely shared recommendations, we believe our analysis provides a more comprehensive, transparent, evidence-based and theoretically informed rationale for analysis of preclinical studies," the authors conclude. 